\title{
Application of Artificial Intelligence in the Field of Power Systems
}

\author{
Xu Jizhi ${ }^{1}$, Zhang Xinyan ${ }^{2}$, Li Jianwei ${ }^{1}$ \\ ${ }^{1}$ School of Electrical Engineering, Xinjiang University, Urumqi, China \\ ${ }^{2}$ Engineering Research Center for Renewable Energy Power Generation and Grid Technology, Education Ministry, Urumqi, China
}

Email address:

339802968@qq.com (Xu Jizhi),xjcxzxy@163.com (Zhang Xinyan),1622209843@qq.com (Li Jianwei)

\section{To cite this article:}

Xu Jizhi, Zhang Xinyan, Li Jianwei. Application of Artificial Intelligence in the Field of Power Systems. Journal of Electrical and Electronic Engineering. Vol. 7, No. 1, 2019, pp. 23-28. doi: 10.11648/j.jeee.20190701.13

Received: January 10, 2019; Accepted: February 27, 2019; Published: March 8, 2019

\begin{abstract}
In recent years, the development of power systems has advanced by leaps and bounds. With the development of artificial intelligence, new directions have emerged. China has upgraded the development of artificial intelligence to a national strategy. A new proportion of new energy terminals is connected to the power grid. Modern power systems present complexity and uncertainty. Artificial intelligence technology will be an effective measure to solve complex system control and decision problems. Based on the application of artificial intelligence in the field of power system application. As a key link in the end-use of energy systems, the degree of intelligence of the power system will greatly affect the smooth implementation of the above technological innovations and advancements. At the same time, the pivotal link of the power system will cause the energy system to focus on the power system and Energy-related technologies are the basis for the integration and integration of the entire energy system. Therefore, it is essential to focus on the development of intelligent technologies in the power system. this paper expounds the application of artificial intelligence technology in power system scheduling, planning and power market.
\end{abstract}

Keywords: Artificial Intelligence, Intelligent Scheduling, New Generation of Electric Power System

\section{Introduction}

Following the automation and information revolution, intelligence has become an important direction of the fourth industrial revolution. Artificial intelligence has triggered widespread attention and industrialization at home and abroad. In 2016, the White House released the National Artificial Intelligence and Development Strategic Plan [1], the UK released "Preparing for the Future of Artificial Intelligence", and the French government issued the "National" in 2017. In the same year, China released the "New Generation Artificial Intelligence Development Plan". Artificial intelligence has been successfully applied in the field of biology [2], medical field [3], image field [4], etc. In the future, artificial intelligence will be deeply integrated with various fields of economy and society, for energy, financial services, urban operation, retail, manufacturing. Major changes have been made in areas such as industry. State Grid Co. Ltd. established the Artificial Intelligence
Application Research Institute and the Artificial Intelligence Joint Laboratory. China Southern Power Grid Corporation and Baidu signed the "Internet + Power" strategic cooperation agreement. Both power grid companies are promoting artificial intelligence technology in the field of smart grid. Development and application.

Based on the comparison of a large number of domestic and foreign literature, this paper introduces the application scenarios and research progress of artificial intelligence in power systems, and puts forward the research prospects. Finally, the limitations and development prospects of artificial intelligence in power systems are discussed.

\section{The Development Status and Trend of Artificial Intelligence}

\subsection{Introduction to Artificial Intelligence}

Artificial intelligence is an interdisciplinary subject that 
combines computer science, control science, physiology, and philosophy. In recent years, the rapid development of artificial intelligence has benefited from massive basic data, hardware equipment innovation, and breakthroughs in core algorithms. The core algorithm includes neural networks, deep learning, migration learning, enhanced learning, and generative confrontation learning algorithms. Typical deep learning models include deep belief nets (DBN) [5], convolution neural network (CNN) [6], and long-short term memory (LSTM) [7], stacked auto encoder (SAE) [8] and so on. The deep learning method does not need to manually participate in feature extraction. Through the layer-by-layer feature transformation, the feature representation of the sample in the original space is transformed into a new feature space, which can further describe the rich intrinsic information of the data, thereby improving the accuracy of classification or prediction. It greatly improves the accuracy and potential of algorithms such as artificial neural networks in the past, and accelerates the pace of artificial intelligence to popular applications. At present, the leading edge of artificial intelligence technology is mainly Internet high-tech enterprises, and many new products and applications have come out. In foreign countries, Google, Microsoft, Facebook, IBM, etc. are pioneers of artificial intelligence. Google has acquired 16 startups including Deep Mind, and is actively deploying algorithms, hardware and products. Facebook uses natural language understanding technology to analyze and understand the user's entire behavior on Facebook, so that users can select the content they are interested in. Watson, a cognitive computing platform developed by IBM, has the ability to understand, reason, learn, and interact, and has been gradually applied to various industries. In China, Internet giants such as Baidu, Alibaba and Tencent have accelerated the deployment of artificial intelligence. Baidu is currently focusing on the field of autonomous driving. Tencent has established the Artificial Intelligence Laboratory in Seattle, USA, and has also launched some applications. For example, more than $80 \%$ of the customer consultations of Tencent's Weizhong Bank are completed by intelligent customer service robots. Alibaba's development of artificial intelligence is more application-oriented, and launched a series of specific scenarios, such as artificial intelligence city brain, medical brain, industrial brain and so on.

\subsection{Artificial Intelligence Development Trend}

At the 2017 Davos Forum, Accenture released the report "Artificial Intelligence: Helping China's Economic Growth". It is expected that by 2035, artificial intelligence is expected to accelerate the annual growth rate of China's economy from $6.3 \%$ to $7.9 \%$, including manufacturing, Agriculture, forestry, fisheries, wholesale and retail are the three industries that have benefited the most. PwC also released a report on artificial intelligence, which is expected to boost global GDP growth by $14 \%$ by 2030 , equivalent to $\$ 15.7$ trillion, contributing $26 \%$ to China's GDP, and the three industries that benefit the most. It is: retail, financial services and health care.

Artificial intelligence has triggered and accelerated the new round of technological revolution and industrial revolution, especially in the traditional manufacturing, retail, and public service industries. Artificial intelligence will bring about major changes in areas with high repetitiveness, strong interactivity, and obvious cross-domain learning characteristics. There are three main areas: 1 automation field, artificial intelligence will give the machine "brain" compared to the traditional execution of the established command, so that it has the ability to autonomously perceive the environment, make independent learning decisions, and perform operations autonomously, such as automatic driving, robots. Logistics, industrial robots, etc. 2 In the consumer field, artificial intelligence will fully upgrade smart products, consumer services, etc. More personalized smart products will be developed, such as car, home, wear, hand-held, etc. and consumer services will become more integrated. 3 In the field of public services, artificial intelligence provides personalized, diversified and high-quality services to the public, such as education, robots, intelligent medical assistants, and medical escort robots. In the future, human beings will benefit more from the transformation of artificial intelligence. Human beings are more responsible for deciding which problems are worth solving and why, artificial intelligence is responsible for solving problems, and artificial intelligence has become a continuation of human wisdom through other means.

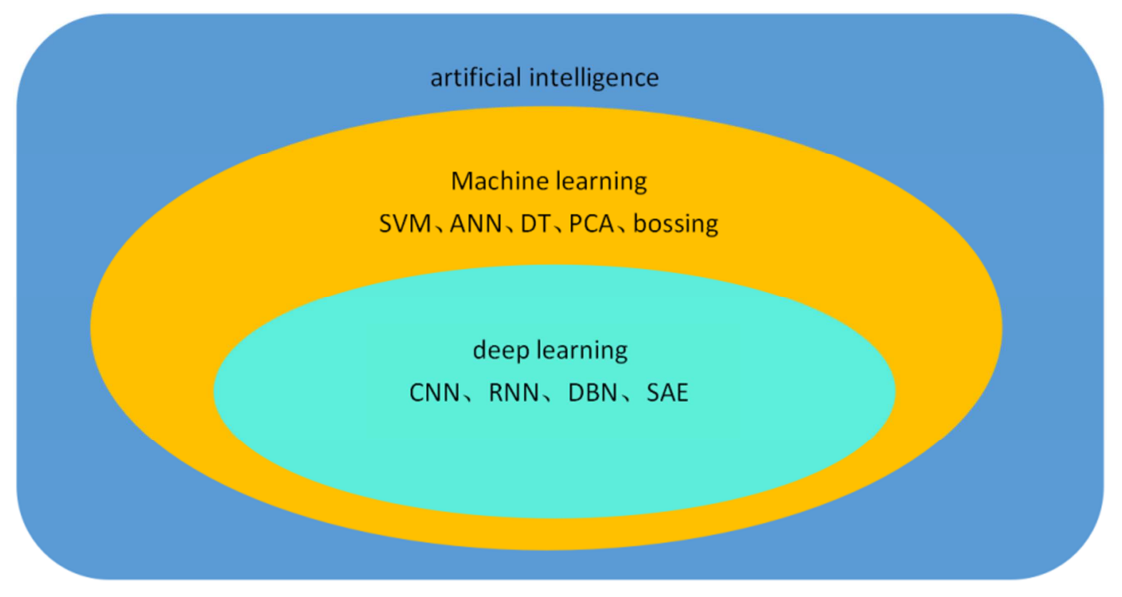

Figure 1. The relationship between artificial intelligence and machine learning. 


\section{Artificial Intelligence and Power System}

\subsection{Artificial Intelligence Application Scenarios in Power Systems}

Electricity is the energy pillar of today's society and the "industrial blood" that rivals oil. With the advancement of society and the development of science and technology, there is a growing desire for stable, high-quality, reliable sources of electricity supply. However, human factors, self-operation problems and natural disasters have invaded this stable supply all the time, causing accidents to occur frequently. How to prevent accidents from happening in advance and ensure stable and continuous supply of electricity is not only a concern of people, but also an urgent problem for people to solve. The application of artificial intelligence in the new generation of power systems is conducive to efficient allocation of resources, greatly reducing operating costs, expanding the breadth and depth of grid services, and forming a highly intelligent grid operation. It has rich application scenarios in the fields of power dispatching, inspection, marketing, intrinsic safety, and operation management.

At present, for the modern power system with large scale and complex structure, the main purpose of scheduling is to ensure the safe operation of the system, ensure the reliability of the power supply of the user, and improve the economics of the system $[9,10,11]$. The fundamental problem is to solve the complex network. There are constrained optimization problems. On the mathematical model, the objective function is set according to the scheduling target and the constraints of the physical operation of the network are satisfied, and the optimal operation scheme is formulated by using the optimization method [12, 13, 14]. The scheduling method based on artificial intelligence technology corresponds to Power system scheduling functions, power system scheduling issues are optimized. Expert systems have been used to solve power system scheduling problems in the 1980s. According to the basic data of power dispatching, the expert system architecture and different heuristic rules are constructed. According to the generator capacity classification, a rough 24-hour scheduling strategy is formulated, and global optimization is realized through logical reasoning [15]. Considering that load fluctuations and access to renewable energy may lead to insufficient unit capacity, non-parametric neural networks are used to quantify the uncertainty caused by wind power, and combined with Monte Carlo method, wind power generation scenarios are taken as The constraint condition is incorporated into the stochastic security constraint combination optimization model, and the optimal combination optimization scheme is solved by genetic algorithm. The simulation method proves that the method reduces the operational risk of the smart grid $[16,17]$.

Accurate and fast intelligent dispatching through artificial intelligence, the continuous development of complex AC/DC power systems and large-scale access of new energy have put forward more rapid and intelligent requirements for power grid operation control. Based on artificial intelligence technology such as deep learning to construct the power grid dispatching brain, it has the characteristics of deep analysis of power grid operation mechanism, self-understanding learning of control procedures, and rapid fault handling. It can analyze the transient steady state operation of the power grid in real time, and automatically identify the instability mechanism of the power grid for rapid event. Link, online generation and adjustment control scheme; self-understanding the text of learning scheduling rules, mining control behavior patterns, automatically generating control tasks, constructing expert rule bases, automatic navigation disposal tasks, etc.; online research and analysis of future power flow and risk monitoring and early warning of large power grids, fast search The key chain fault path of the UHV grid, autonomously isolates the grid fault, reconstructs the grid topology, and maximizes the grid security and power supply reliability level. Artificial intelligence technology can help solve nonlinear power system problems, the application of artificial neural network in relay protection, the application of artificial intelligence algorithm in power system operation, the application of fuzzy theory in power system operation and the expert system in power The application in which the system is running. In the power system, the expert system is most widely used in fault diagnosis and recovery processing. The expert system is effectively distinguished through knowledge representation and reasoning, which provides convenience for the development of related work. The knowledge base of the expert system with fault diagnosis is formed, and then the knowledge base content is further inferred according to the received alarm information to obtain the result of correct diagnosis of the fault. Although the expert system is a widely used fault diagnosis method, the fault diagnosis expert system needs to establish a device fault model in advance, and can only perform forward reasoning, but cannot perform backward reasoning and hybrid reasoning, and obtain possible faulty equipment. The large number is not conducive to the rapid confirmation of faulty equipment. If the actual fault does not match the model, the correct result will not be obtained, so there are still many limitations.

In the inspection and inspection of the power system, intelligent inspection robots and drones can realize standardized and intelligent operations, improving efficiency and safety. The intelligent inspection robot is equipped with a variety of detectors, which can observe the equipment at close range, and the accuracy of the inspection is high [18]. In the power system fault diagnosis, the traditional method increases the complexity and uncertainty of the method based on artificial feature extraction and empirical judgment. When dealing with complex fault information and fault diagnosis of large networks, its scope of application is limited. The deep learning method can deeply learn the structural features 
inherent in the data, and integrate the learned feature information into the model establishment process, thereby reducing the inadequacy of artificial design features and the complexity brought by traditional feature extraction.

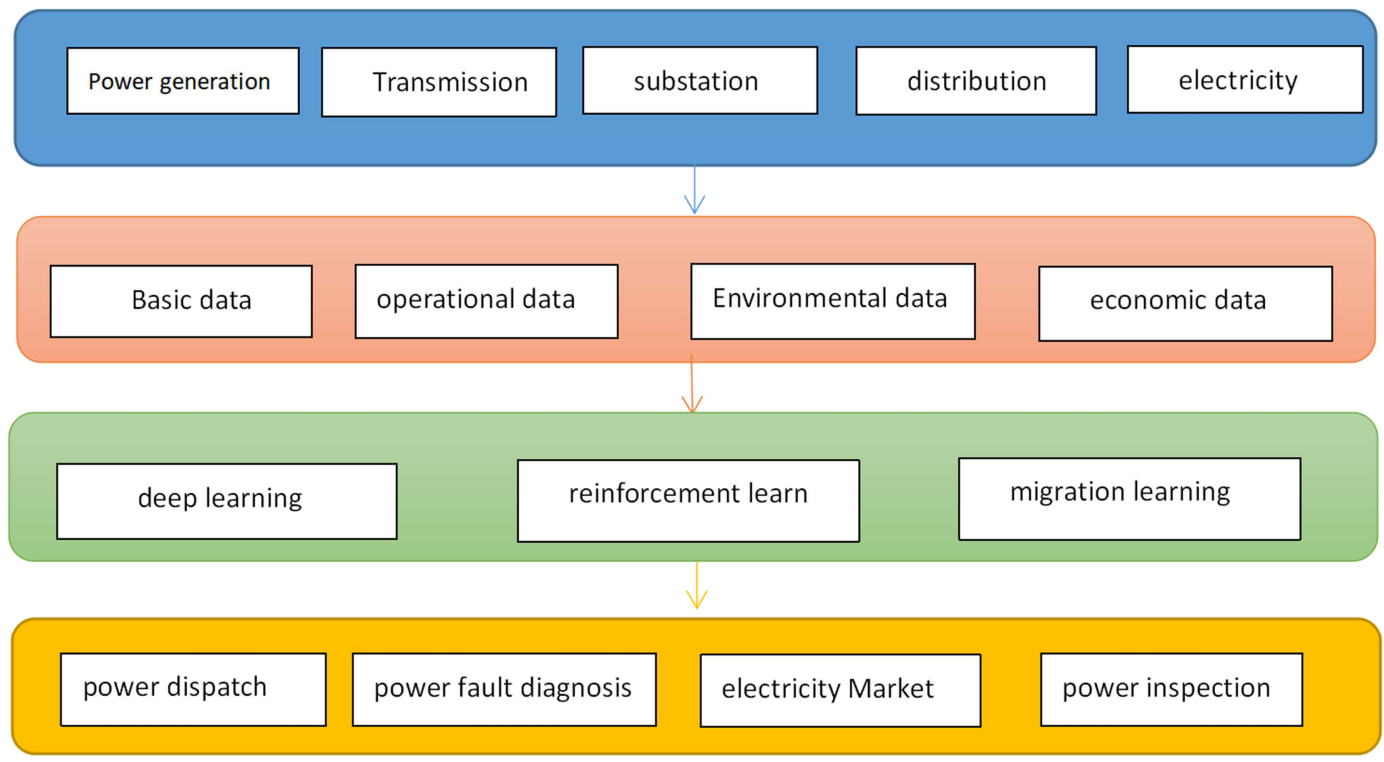

Figure 2. The application framework of new generation artificial intelligence in smart grids.

\subsection{The Development Trend of Artificial Intelligence in Power System}

The combination of artificial intelligence and power system is the trend of future research in the field of power systems, which is to maintain the operation of power systems, reduce the occurrence of accidents, equipment management and investment return analysis, grid systemic risk assessment and forecasting, client analysis, New energy production and consumption patterns have significant progress. The development of a new generation of power systems should be accompanied by the stability, synchronization and long-term development of artificial intelligence technology. Based on ubiquitous sensing technology, artificial intelligence technology is maturing based on emerging technologies such as deep learning, the Internet of Things and big data block chain. In the future, a new generation of power systems will eventually be intelligent power systems, and AI technology will be spread across power system equipment management and system control, energy management and trading. When the input and output data of the power system has many dimensions and correlations, it is difficult to obtain a comprehensive control and optimization strategy. Deep learning and reinforcement learning theory are combined and the basic framework of knowledge migration is constructed. The gridded knowledge migration learning algorithm is proposed to realize information extraction, which can greatly optimize the power system $[19,20]$.

The application in power system planning is mainly reflected in power supply planning, power grid planning and so on [21]. 1 In the aspect of power supply planning, a hybrid artificial intelligence algorithm is proposed for substation planning in urban power grid planning [22]. The algorithm quickly determines the number of new substations to be built and the initial feasible solutions for the capacity of each substation through the core algorithm. Hopfield neural network to check the power supply range and new site of the substation. The Hopfield neural network can reduce the value of the energy function to a minimum, which has a good effect on solving such complex optimization problems. 2 In terms of power grid planning, the construction plan of the transmission line that optimizes the economic benefits of the transmission system is determined based on the load forecasting and power supply planning under the transmission requirements of the planning period. Continue to deepen the intelligent replacement of simple business in the power system, and cultivate a team of talents who are both familiar with the business and understand artificial intelligence technology, and build artificial intelligence basic experiments and public service platforms. Through artificial intelligence technology, intelligent analysis and decision-making of complex problems in professional fields are gradually realized, and artificial intelligence systems capable of handling business in professional fields are gradually established. Most of the grid business has a high level of intelligence, but the businesses are often independent of each other and fail to fully play an effective synergy. The use of artificial intelligence technology to effectively integrate the various systems, play a synergistic effect between the systems, maximize the potential value of the system, and achieve management optimization. Under the pattern of the development of the grid to the energy Internet and the wide-area interconnection of high-voltage and large-scale power grids, the integration of artificial intelligence and grid application technology will effectively improve the ability to control complex power grids, improve the security of grid operations, and transform the grid service service model. The ultimate goal of artificial intelligence is to achieve universal intelligence that is similar to humans with 
abstract thinking and creative thinking. Throughout the historical process, the development of artificial intelligence is still in the stage of transition from perceived intelligence to cognitive intelligence. There is still a long way to go before it can develop into an artificial intelligence system with thinking, creativity and emotional ability. After years of development and innovation, artificial intelligence technology has become a hot research and application technology in the new era, and its role has been extended to all areas of society. We also need the help of artificial intelligence in the construction of smart grid, but artificial intelligence also has certain problems such as safety, different application environment, stability and other risks, so artificial intelligence technology should be used reasonably in the construction of smart grid. The application of artificial intelligence technology can solve many traditional problems. Perfect artificial intelligence technology can better improve the intelligent level of the power grid. At the same time, artificial intelligence technology also provides a more comprehensive and safe solution for smart grid construction. With the help of smart grid construction will achieve better development.

\section{Conclusion}

Smart grid has become the main development direction of China's power system in the future. China's understanding of smart grid is to integrate new energy, new materials, new equipment and advanced information technology, control technology energy storage technology to achieve digital management of power generation, transmission, distribution, use and storage, intelligent decision-making Interactive transactions, optimize resource allocation, meet the diversified power needs of users, ensure the safety, reliability and economy of power supply, meet environmental constraints, and adapt to the development needs of power market. Smart grids should be characterized by openness, security, efficiency, cleanliness and self-healing. In the development of smart grid, whether it is digital management, intelligent decision-making or interactive transactions, the optimization process is inseparable. Optimization is an essential element in the implementation of smart grids. Although there have been many literature using artificial intelligence algorithms to do a lot of optimization work on all aspects of the power system, but basically have a single optimization goal, the algorithm is applied with blunt features, both in terms of optimization speed and optimization results, there is a great improvement. space.

Artificial intelligence provides unlimited possibilities for the development of power systems. In the future, the structure and operation mode of power grids will undergo major changes. New material technologies will be widely used in power grids, and physical grids will be highly integrated with information systems. In the promotion of artificial intelligence development and application, it has advantages in data, capital, application scenarios, etc., but currently there are shortcomings in hardware, basic algorithms, and talents. This is also the bottleneck for the development of artificial intelligence in China. Grid enterprises should develop artificial intelligence. Implementing the most cutting-edge theory, technology and tools, focusing on the application of the project.

\section{References}

[1] SUN Bolin. New report on artificial intelligence in the United States and its implications for Us $[\mathrm{J}]$. Techniques of Automation \& Applications, 2017, 36 (10): 1-7.

[2] MAHMUD M, KAISER M S, HUSSAIN A, et al. Applications of deep learning and reinforcement learning to biological data $[\mathrm{J}]$. IEEE Transactions on Neural Networks \& Learning Systems, 2018, 29 (6): 2063-2079.

[3] MCBEE M P, AWAN O A, COLUCCI A T, et al. Deep learning in radiology [J]. Academic Radiology, 20181-9.

[4] LITJENS G, KOOI T, BEJNORDI B E, et al. A survey on deep learning in medical image analysis [J]. Medical Image Analysis, 2017, 42 [9]: 60-88.

[5] HINTON G E, OSINDERO S, TEH Y W. A fast learning algorithm for deep belief nets [J]. Neural Computation, 2006, 18 (7): $1527-1554$.

[6] HU B, LU Z, LI H, et al. Convolutional neural network architectures for matching natural language sentences [J]. Advances in Neural Information Processing Systems, 2015, 3: 2042-2050.

[7] ZHU Qiaomu, LI Hongyi, WANG Ziqi, et al. Short-term wind power forecasting based on LSTM [J]. Power System Technology, 2017, 41 (12): 3797-3802.

[8] GEH R ING J, MIAO Y, METZE F, et al. Extracting deep bottleneck features using stacked auto-encoders [C] / / IEEE International Conference on Acoustics, Speech and Signal Processing. IEEE, 2013: 3377-3381.

[9] Ali Y, Rasheed Z, Muhammad N, et al. Energy optimization in the wake of China pakistan economic corridor (CPEC) [J]. J of Control and Decision, 2018, 5 (2): 129-147.

[10] Kopsidas K, Kapetanaki A, Levi V, et al. Optimal demand response scheduling with real time thermal ratings of overhead lines for improved network reliability [J]. IEEE Trans on Smart Grid, 2017, 8 (6): 2813-2825.

[11] Sun Q Y, Teng F, Zhang H G. Energy internet and its key control issues [J]. Acta Automatica Sinica, 2017, 43 (2): 176-194.

[12] Takeuchi A, Hayashi T, Nozaki Y, et al. Optimal scheduling using meta heuristics for energy networks [J]. IEEE Trans on Smart Grid, 2012, 3 (2): 968-974.

[13] Sousa T, Morais H, Vale Z, et al. Intelligent energy resource management considering vehicle-to-grid: A simulated annealing approach [J]. IEEE Trans on Smart Grid, 2012, 3 (1): 535-542.

[14] Zhao B, Guo C X, Cao Y J. A multiagent-based particle swarm optimization approach for optimal reactive power dispatch [J]. IEEE Trans on Power Systems, 2005, 20 (2): 1070-1078. 
[15] Li S, Shahidehpour S M, Wang C. Promoting the application of expert systems in short-term unit commitment [J]. IEEE Trans on Power Systems, 1993, 8 (1): 286-292.

[16] Kurban M, Filik U B. Unit commitment scheduling by using the auto regressive and artificial neural network models based short-term load forecasting [C]. Int Confon Probabilistic Methods Applied To Power Systems. RinCon: IEEE, 2008: $1-5$.

[17] Quan H, Srinivasan D, Khosravi A. Incorporating wind power forecast uncertainties into stochastic unit commitment using neural network-based prediction intervals [J]. IEEE Trans on Neural Networks and Learning Systems, 2015, 26 (9): 2123-2135.

[18] LU Shouyin, ZHANG Ying, LI Jianxiang, et al. Application of mobile robot in high voltage substation [J]. High Voltage Engineering, 2017, 43 (1): 276-284.
[19] LIU Wei, ZHANG Dongxia, WANG Xinying, et al. A decision making strategy for generating unit tripping under emergency circumstances based on deep reinforcement learning [J]. Proceedings of the CSEE, 2018, 38 (1): 109-119, 347.

[20] JIANG Haorong, XU M aoxin, WANG Keying. Grid know ledge transfer learning algorithm and its application in carbon-energy combined-flow optimization [J]. Electric Pow er Construction, 2017, 38 (7): 96-105.

[21] Tan W S, Hassan M Y, Majid M S, et al. Optimal distributed renewable generation planning: A review of different approaches [J]. Renewable and Sustainable Energy Reviews, 2013, 18 (2): 626-645.

[22] Gao W X, Luo X J, Zhu Y. A new distribution substation planning algorithm based on greedy algorithm and Hopfield neural network [J]. Power System Technology, 2004, 28 (7): 73-76. 